\title{
Faint dark matter annihilation signals and the Milky Way's supermassive black hole
}

\author{
Barry T. Chiang $\oplus^{*}$ Stuart L. Shapiro, ${ }^{\dagger}$ and Jessie Shelton $\oplus^{*}$ \\ Departments of Physics and Astronomy, University of Illinois at Urbana-Champaign, \\ Urbana, Illinois 61801, USA
}

(Received 24 January 2020; accepted 7 July 2020; published 24 July 2020)

\begin{abstract}
A wide range of mechanisms predict present-day $s$-wave dark matter (DM) annihilation cross sections that are orders of magnitude below current experimental sensitivity. We explore the capability of DM density spikes around the Milky Way's supermassive black hole to probe such faint signals of DM annihilations, considering a range of possible spike and halo distributions. As an exemplar of a theory with a suppressed $s$-wave annihilation cross section, we consider a hidden sector axion portal model of DM. In this model, the leading contribution to the annihilation cross section in the early Universe is $p$-wave, while $s$-wave annihilations occur at higher order in the coupling constant. We provide a unified treatment of DM freeze-out in this model including both $s$ - and $p$-wave annihilations and analytically determine the photon spectrum for the dominant DM annihilation process in the Universe today. We find that Fermi and H.E.S.S. observations of the Galactic center offer excellent sensitivity to this model over a wide range of parameter space, with prospects depending sensitively on the properties of the DM spike as well as the central halo.
\end{abstract}

DOI: 10.1103/PhysRevD.102.023030

\section{INTRODUCTION}

The indirect detection of dark matter through its annihilation products in cosmic rays is a cornerstone of the experimental search for dark matter (DM). Indirect detection is an increasingly potent probe of annihilating DM, with observations of (e.g.) both the cosmic microwave background and dwarf galaxies now sensitive to DM with annihilation rates at or below the standard thermal target $\sigma_{\text {thermal }}=3 \times 10^{-26} \mathrm{~cm}^{3} / \mathrm{s}$ in an expanding range of masses and annihilation channels [1-4].

Identifying the annihilation products of $\mathrm{TeV}$-scale $\mathrm{DM}$ with standard thermal-scale cross sections remains a steep observational challenge, however, as the flux of cosmic rays from DM annihilation in galaxy halos falls off with increasing DM mass as $\mathrm{m}^{-2}$. Moreover, many dark matter models predict present-day DM annihilation cross sections substantially below the thermal target. There are many mechanisms that predict a suppressed present-day DM annihilation cross section. For instance, the DM may

\footnotetext{
tchiang5@illinois.edu

slshapir@illinois.edu

sheltonj@illinois.edu
}

Published by the American Physical Society under the terms of the Creative Commons Attribution 4.0 International license. Further distribution of this work must maintain attribution to the author(s) and the published article's title, journal citation, and DOI. Funded by SCOAP ${ }^{3}$. simply arise from a dark sector that is very cold compared to the Standard Model (SM) [5,6]; it may have been diluted by entropy production post-freeze-out [7-9]; its annihilation cross section may be strongly velocity-dependent, because of kinematics [10-13] or symmetries [14-18] (or both); or its annihilation cross section in the early Universe may involve additional species that are later depleted [10,19-22]. In several of these models, e.g., [5,6,17], terrestrial signals are typically significantly reduced compared to expectations from thermal WIMPs, making even a suppressed indirect detection signal an irreplaceable discovery handle and a powerful window onto the physics of DM.

Here we estimate the sensitivity to faint $s$-wave DM annihilation cross sections that can potentially be offered by DM density spikes around the supermassive black hole (SMBH) at the center of the Milky Way. Black holes focus DM within their gravitational zone of influence into a steep, localized overdensity known as a spike [23]. This enhancement of the DM density can potentially magnify DM annihilation rates by many orders of magnitude in the immediate vicinity of the black hole, leading to a bright, pointlike source of cosmic rays. While this enormous magnification of DM annihilation signals can offer a uniquely powerful window onto models of DM with suppressed annihilation cross sections and thus suppressed signatures in DM haloes [17,24-27], the details of the predicted spike distribution depend sensitively on as-yetunknown properties of the host DM halo, the central stellar 
cusp within that halo, and the formation history of the black hole [23,28-33]. Accordingly, we consider a broad range of possible DM distributions in the Galactic center in this work, with the aim of understanding what parameter ranges offer interesting sensitivity to subthermal annihilation cross sections. Uncertainties in the spike distribution translate into very large uncertainties in possible DM signal strengths, making it hard to unambiguously constrain DM models using annihilation signals within DM spikes. Nonetheless DM spikes can provide an invaluable potential opportunity for discovery, particularly for models with suppressed annihilation cross sections and sharp spectral features.

As a representative DM model with both challenging annihilation cross sections and sharp spectral features, we consider the hidden sector axion portal (HSAP) model developed in [17,27]. In this model, fermionic DM $\chi$ annihilates to pseudoscalars $a$, which subsequently decay to SM electroweak gauge bosons. This model features an interesting interplay of two annihilation channels: the process $\chi \chi \rightarrow a a$ proceeds in the $p$-wave, while the reaction $\chi \chi \rightarrow a a a$ contributes in the $s$-wave when it is kinematically available, but is higher order in the coupling constant. For low DM masses, the $p$-wave process dominates DM annihilation in the early Universe, yielding a subdominant $s$-wave annihilation cross section orders of magnitude below the standard thermal target. For high DM masses, the $s$-wave annihilation process can dominate during thermal freeze-out, resulting in thermal-scale cross sections but signals that are observationally challenging thanks to the large DM mass. In all cases the $s$-wave process $\chi \chi \rightarrow$ aaa dominates the present-day DM annihilation rate, including within DM spikes.

We begin in Sec. II with an overview of the HSAP model, including a novel treatment of freeze-out incorporating both $s$ - and $p$-wave contributions to the annihilation cross section, and a calculation of the photon spectrum from the resulting DM annihilations. In Sec. III we detail our model of DM density spikes around the Milky Way's SMBH. We compare predicted gamma-ray fluxes from DM annihilation within SMBH-induced density spikes to observations from Fermi and H.E.S.S. in Sec. IV and discuss the resulting prospects for sensitivity, and in Sec. V we conclude.

\section{THE PARTICLE MODEL}

In this section we discuss a hidden sector axion portal model of dark matter, as introduced in [17]. In this model, DM is a Majorana fermion $\chi$ which annihilates to pseudoscalars $a$, which subsequently decay to the SM via axionlike couplings to SM gauge bosons. This model is $C P$-conserving, ensuring that the leading annihilation process $\chi \chi \rightarrow a a$ is $p$-wave. In contrast to previous works $[17,27]$, we will focus on the regime where the higher-order but $s$-wave annihilation process $\chi \chi \rightarrow a a a$ is kinematically available, and explore the consequences both for thermal freeze-out and for potential $\mathrm{BH}$ spike signals in our Galaxy today.

\section{A. Annihilations and relic abundance}

The hidden sector axion portal (HSAP) model is described by the Lagrangian,

$\mathcal{L}=\bar{\chi}\left(i \gamma \cdot \partial-m_{\chi}\right) \chi-\frac{1}{2}(\partial a)^{2}-\frac{1}{2} m_{a}^{2} a^{2}-i y a \bar{\chi} \gamma^{5} \chi$.

This Lagrangian has three free parameters: the masses $m_{\chi}$ and $m_{a}$, and the Yukawa coupling constant $y$, which can be determined in terms of $m_{\chi}$ and $m_{a}$ using the requirement that thermal freeze-out of DM annihilations yields the observed DM relic abundance. Additionally, the mediator $a$ is coupled to the SM via dimension-five axionlike interactions with SM gauge fields, which enables it to decay promptly on astrophysical scales, as we discuss further below.

\section{Annihilation cross sections}

The leading $2 \rightarrow 2$ annihilation process occurs in the $p$ wave, with thermally averaged cross section given in the nonrelativistic limit by [17]

$$
\langle\sigma v\rangle_{p}=\frac{1}{x} \frac{y^{4}}{4 \pi m_{\chi}^{2}} \sqrt{1-\eta^{2}} \frac{\left(1-\eta^{2}\right)^{2}}{\left(2-\eta^{2}\right)^{4}}
$$

where

$$
\eta \equiv m_{a} / m_{\chi}
$$

and

$$
x \equiv m_{\chi} / T .
$$

The velocity dispersion is related to the temperature as $\left\langle v^{2}\right\rangle=6 / x$.

There is one Feynman diagram topology for the process $\chi \chi \rightarrow a a a$, shown in Fig. 1, which yields six distinct diagrams thanks to the permutation of final state momenta among the identical particles. The calculation of the matrix element simplifies significantly in the nonrelativistic limit, where all of the angular dependence drops out. The resulting expression for the spin-averaged matrix element is
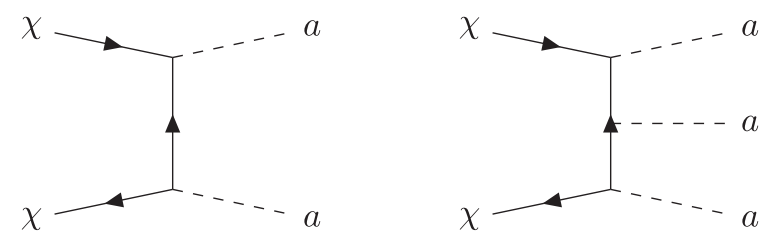

FIG. 1. Representative Feynman diagrams for $p$-wave (left) and $s$-wave (right) DM annihilation processes. 


$$
|\overline{\mathcal{M}}|^{2}=\frac{16 y^{6}}{m_{\chi}^{2}} f\left(w_{1}, w_{2} ; \eta\right),
$$

where the variables $w_{i}$ parametrize the distribution of energy among the three final state particles in the center of mass frame,

$$
w_{i} \equiv \frac{E_{i}}{E_{\chi}}, \quad \sum_{i=1}^{3} w_{i}=2,
$$

and

$f\left(w_{1}, w_{2} ; \eta\right) \equiv\left[\frac{\left(1-\frac{\eta^{2}}{4}\right)\left(1+\frac{3 \eta^{2}}{4}\right)+\sum_{i=1}^{3}\left(\frac{1}{2} w_{i}^{2}-w_{i}\right)}{\prod_{i=1}^{3}\left(w_{i}-\frac{\eta^{2}}{2}\right)}\right]^{2}$.

In the nonrelativistic limit, the cross section for $\chi \chi \rightarrow a a a$ is then

$$
\sigma v=\frac{y^{6}}{96 \pi^{3} m_{\chi}^{2}} \int d w_{1} d w_{2} f\left(w_{1}, w_{2} ; \eta\right)+\mathcal{O}\left(v^{2}\right),
$$

where the upper and lower limits of integration for $w_{2}$ are given by

$$
\begin{aligned}
w_{2 \pm} & =1-\frac{\eta^{2}}{4}-2 \varepsilon_{j} \varepsilon_{k} \pm \frac{1}{2} \sqrt{\left(4 \varepsilon_{j}^{2}-\eta^{2}\right)\left(4 \varepsilon_{k}^{2}-\eta^{2}\right)}, \\
\varepsilon_{j} & =\frac{1}{2} \sqrt{1-w_{1}+\frac{\eta^{2}}{4}}, \\
\varepsilon_{k} & =\frac{1}{2} \frac{w_{1}-\frac{\eta^{2}}{2}}{\sqrt{1-w_{1}+\frac{\eta^{2}}{4}}},
\end{aligned}
$$

and for $w_{1}$ the upper and lower limits are

$$
w_{1-}=\eta, \quad w_{1+}=1-\frac{3}{4} \eta^{2} .
$$

The velocity-dependent $\mathcal{O}\left(v^{2}\right)$ term is negligible both at freeze-out (where it is higher order in $y^{2} / 4 \pi$ compared to the contribution from $\chi \chi \rightarrow a a$ ) and in the Milky Way today. We thus retain only the piece of Eq. (8) that is constant as $\left\langle v^{2}\right\rangle \rightarrow 0$, defining a contribution to the $s$-wave annihilation $\langle\sigma v\rangle_{s}$.

\section{Thermal freeze-out and relic abundance}

To determine the Yukawa coupling constant $y$, we include the leading contributions to both $s$ - and $p$-wave annihilation processes and solve the Boltzmann equation governing the DM relic abundance. In the nonrelativistic limit, this Boltzmann equation can be written as

$$
\frac{d Y}{d x}=-\lambda x^{-2}\left(\langle\sigma v\rangle_{s}+\langle\sigma v\rangle_{p}\right)\left(Y^{2}-Y_{\mathrm{eq}}^{2}\right)
$$

where $\lambda=0.264\left(g_{* S} / g_{*}^{1 / 2}\right) m_{\mathrm{Pl}} m_{\chi}$ and the equilibrium yield is $Y_{\text {eq }}=0.29\left(g_{* S}\right)^{-1} x^{3 / 2} e^{-x}$ [34]. Here $m_{\mathrm{Pl}}=G^{-1 / 2}$ is the Planck mass, and $g_{*}$ and $g_{* S}$ are the number of effective relativistic degrees of freedom contributing to the energy and entropy densities, respectively. The present-day DM relic abundance is taken to be $\Omega_{\mathrm{DM}} h^{2}=0.112$ [1].

Figure 2 shows the resulting contours of $y^{2} / 4 \pi$ in the $m_{\chi}-\eta$ parameter space. The model becomes nonperturbative for $y^{2} / 4 \pi \gtrsim 1$, which restricts $m_{\chi} \lesssim 14 \mathrm{TeV}$. The interplay between $s$ - and $p$-wave contributions during freeze-out becomes especially important for heavier $m_{\chi}$ and smaller $\eta$, where the $\chi \chi \rightarrow a a a$ annihilation is less suppressed compared to the $\chi \chi \rightarrow a a$ process.

Figure 3 compares the $s$-wave (solid) and $p$-wave (dashed) annihilation cross sections for four representative values of the mass ratio, $\eta=0.05$ (blue), 0.3 (yellow), 0.6 (green), and 0.66 (red). In this figure we evaluate the

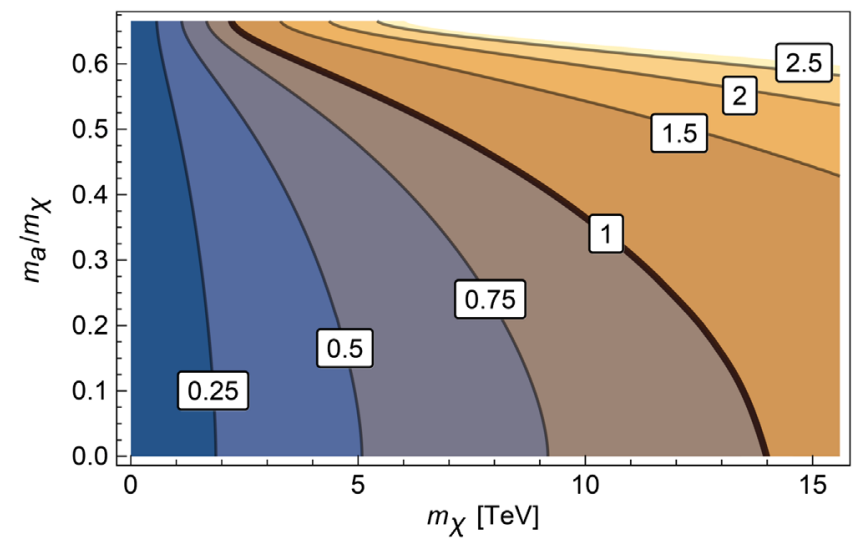

FIG. 2. Value of $y^{2} / 4 \pi$ yielding the correct DM relic abundance as a function of $m_{\chi}$ and $\eta=m_{a} / m_{\chi}$. The thick black contour indicates $y^{2} / 4 \pi=1$, beyond which the model becomes nonperturbative.

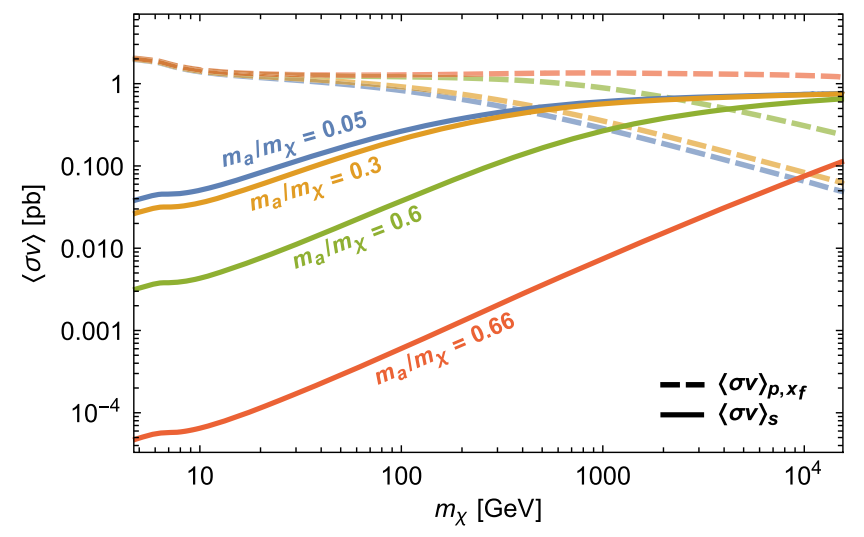

FIG. 3. Thermal $s$-wave (solid) and $p$-wave (dashed) annihilation cross sections, evaluated at $x_{f}$, for $\eta=0.05$ (blue), 0.3 (yellow), 0.6 (green), and 0.66 (red). The $s$-wave annihilation contribution becomes appreciable at thermal freeze-out for large $m_{\chi}$. 
$p$-wave annihilation cross section at freeze-out, $x_{f}$, as determined through the sudden freeze-out condition,

$$
n\left(x_{f}\right)\left(\langle\sigma v\rangle_{s}+\langle\sigma v\rangle_{p}\left(x_{f}\right)\right) \approx H\left(x_{f}\right) .
$$

Here the Hubble rate is given by $H\left(x_{f}\right)=1.66 \sqrt{g_{*}} m_{\chi}^{2} / m_{\mathrm{P} 1} x_{f}^{-2}$. The resulting values of $x_{f}$ range from $22<x_{f}<32$ for $5 \mathrm{GeV}<m_{\chi}<14 \mathrm{TeV}$ and $0<\eta<2 / 3$. Since $x_{f}$ increases with increasing $m_{\chi}$, at large $m_{\chi}$ the $s$-wave cross section becomes increasingly important at $x_{f}$ compared to the velocity-suppressed $p$-wave contribution and can dominate freeze-out for sufficiently heavy DM and sufficiently small $\eta$. The size of the $s$-wave cross section depends sensitively on the mass ratio $\eta$ and is strongly suppressed as $\eta$ approaches the kinematic limit of $2 / 3$.

Since the typical DM velocity dispersion in the Galaxy today is $\left\langle v^{2}\right\rangle=\mathcal{O}\left(10^{-6}\right)$, whereas $\left\langle v^{2}\right\rangle=\mathcal{O}\left(10^{-1}\right)$ at $x_{f}$, the $p$-wave cross section today is suppressed by 5 to 6 orders of magnitude relative to its value at freeze-out. Figure 3 thus demonstrates that DM annihilations in the Galaxy today are dominated by the $s$-wave $\chi \chi \rightarrow$ aaa process. We observe two distinct regimes, depending on whether the $p$ - or $s$-wave process dominates at $x_{f}$. When the $p$-wave process dominates, at small DM mass and large $\eta$, the $s$-wave annihilation cross section is suppressed by orders of magnitude compared to the typical thermal target $(\sim 1 \mathrm{pb})$. On the other hand, when the $s$-wave cross section dominates, at large DM mass and small $\eta$, it is comparable to the thermal target.

\section{Pseudoscalar decays into SM final states}

The pseudoscalar $a$ can decay to the SM through dimension-five axionlike couplings to SM gauge bosons. For simplicity, we will consider here the case when the pseudoscalar couples at leading order only to the hypercharge field strength $B^{\mu \nu}$ via the interaction,

$$
\frac{a}{\Lambda} \epsilon_{\mu \nu \rho \sigma} B^{\mu \nu} B^{\rho \sigma}
$$

This choice is also an interesting scenario for discoverability, as it yields an energetic gamma-ray spectrum with a distinctive spectral feature.

After electroweak symmetry breaking, the interaction of Eq. (13) mediates the decays $a \rightarrow \gamma \gamma$ and, if kinematically possible, $a \rightarrow Z \gamma$ and $a \rightarrow Z Z$. For a given value of $m_{a}$, the partial widths into $\gamma \gamma, Z \gamma$ and $Z Z$ final states following from the interaction of Eq. (13) are given by

$$
\begin{aligned}
\Gamma(a \rightarrow \gamma \gamma) & =\frac{C}{2} \cos ^{2} \theta_{W}, \\
\Gamma(a \rightarrow Z \gamma) & =2 C \cos \theta_{W} \sin \theta_{W}\left(1-\frac{m_{Z}^{2}}{m_{a}^{2}}\right)^{3} \Theta\left(m_{a}-m_{Z}\right), \\
\Gamma(a \rightarrow Z Z) & =\frac{C}{2} \sin ^{2} \theta_{W}\left(1-\frac{4 m_{Z}^{2}}{m_{a}^{2}}\right)^{3 / 2} \Theta\left(m_{a}-2 m_{Z}\right),
\end{aligned}
$$

where $\theta_{W}$ is the Weinberg angle and $C \sim m_{a} / \Lambda^{2}$ is a common constant of proportionality. We require $C$ to be small enough that annihilations of DM directly to SM gauge bosons through an intermediate $a$ are negligible in comparison to the secluded annihilation processes of Fig. 1, but otherwise our results do not depend on $C$. We require only the relative branching fractions of the pseudoscalar into the various decay channels that follow from Eq. (14).

Finally, it is worth observing that, unlike traditional WIMP models, the final states in this model are dominated by photons. In theories that produce copious amounts of charged particles in DM annihilations, secondary synchrotron radiation can provide a competitive probe of DM annihilations within a spike, thanks in large part to the better angular resolution afforded by the lower-energy photons [35-37], although the relative magnitude of this synchrotron signal depends on relatively uncertain aspects of the modeling of the Galactic center [38]. However, in the nightmare dark matter model studied here, DM annihilations proceed directly to gamma rays in the mass range $m_{\chi} \sim \mathcal{O}(100 \mathrm{GeV})$ where radio constraints are especially relevant. Thus the primary signal of this model is in gamma rays, where the photon spectrum exhibits a prominent and distinctive feature, as we discuss next.

\section{B. Photon spectra}

In this subsection we determine the photon spectra $d N / d E_{\gamma}$ resulting from DM annihilations $\chi \chi \rightarrow a a a$.

\section{Photon spectra from $a \rightarrow \gamma \gamma$ decays}

We begin with the photon spectrum $d N / d E_{\gamma}$ from an $s$ wave annihilation process $\chi \chi \rightarrow a a a \rightarrow 6 \gamma$, which can be obtained analytically in the nonrelativistic limit. Consider an individual $a$ particle with energy $E_{a}$ in the Galactic frame decaying into two photons. The maximum and minimum energies the photons can have are

$$
E_{\max / \min }=\frac{1}{2}\left(E_{a} \pm \sqrt{E_{a}^{2}-m_{a}^{2}}\right) .
$$

As the decays of $a$ are isotropic in its rest frame, the energy distribution of daughter photons is uniform between the kinematic boundaries. Defining the dimensionless variables,

$$
u \equiv \frac{E_{\gamma}}{m_{\chi}}=\frac{E_{\gamma}}{E_{a}} \frac{E_{a}}{m_{\chi}},
$$

and $w \equiv E_{a} / m_{\chi}$, the kinematic end points can be written

$$
u_{\max / \min }=\frac{1}{2}\left(w \pm \sqrt{w^{2}-\eta^{2}}\right) .
$$

For a given $w$ and $\eta$, the probability $P(u ; w, \eta) d u$ of finding a photon within the energy interval $d u$ is thus 


$$
P(u ; w, \eta) d u=\frac{d u}{\left(u_{\max }-u_{\min }\right)}=\frac{d u}{\sqrt{w^{2}-\eta^{2}}},
$$

as the probability is uniform and unit-normalized over the kinematically allowed interval. The probability of obtaining an $a$ particle with (relative) energy $w$ from the process $\chi \chi \rightarrow a a a$ is given by

$$
\begin{aligned}
P(w ; \eta)=\frac{1}{\sigma} \frac{d \sigma}{d w} & =A \int_{w_{2-}}^{w_{2+}} d w_{2} f\left(w, w_{2} ; \eta\right) \\
& \equiv A f(w ; \eta),
\end{aligned}
$$

where the cross section $\sigma$ for $\chi \chi \rightarrow a a a$ is given in Eq. (8) and $A$ is a normalization factor. The probability density of finding any specific combination of $u, w$ is then

$$
P(u, w ; \eta)=A \frac{f(w ; \eta)}{\sqrt{w^{2}-\eta^{2}}}
$$

As this expression indicates, a daughter photon with energy $u$ may have come from a parent $a$ with a range of possible energies $w$. To obtain the probability of observing a photon with energy $u$, we integrate $P(u, w ; \eta)$ over the range of $w$ consistent with the value of $u$. The maximum possible value of $w$ is, from Eq. (10), $w_{\max }=1-\frac{3}{4} \eta^{2}$, independent of $u$. For a fixed value of $u, w_{\min }$ can be determined from Eq. (17), which gives

$$
w_{\min }(u)=\frac{4 u^{2}+\eta^{2}}{4 u} .
$$

The desired photon spectrum is therefore

$$
\frac{d N}{d E_{\gamma}}(u, \eta)=6 A \int_{w_{\min }}^{w_{\max }} \frac{w_{\max }(\eta)}{w_{\min }(u, \eta)} \frac{f(w ; \eta)}{\sqrt{w^{2}-\eta^{2}}} d w .
$$

The factor of 6 appears here since there are six final state photons in the annihilation $\chi \chi \rightarrow a a a \rightarrow 6 \gamma$. Accordingly, we evaluate the normalization factor $A$ by requiring

$$
\int_{u_{\min }}^{u_{\max }} \frac{d N}{d E_{\gamma}}(u, \eta) d E_{\gamma}=6
$$

In Fig. 4, the normalized photon spectra, weighted by $u^{2}$, are plotted for four different values of $\eta$. For small $\eta$, the energy distribution is broad and peaks at relatively high energies compared to the spectra for larger values of $\eta$. As $\eta$ approaches the kinematic limit of $2 / 3$, the changes in the spectrum shape become increasingly rapid as the available phase space shrinks.

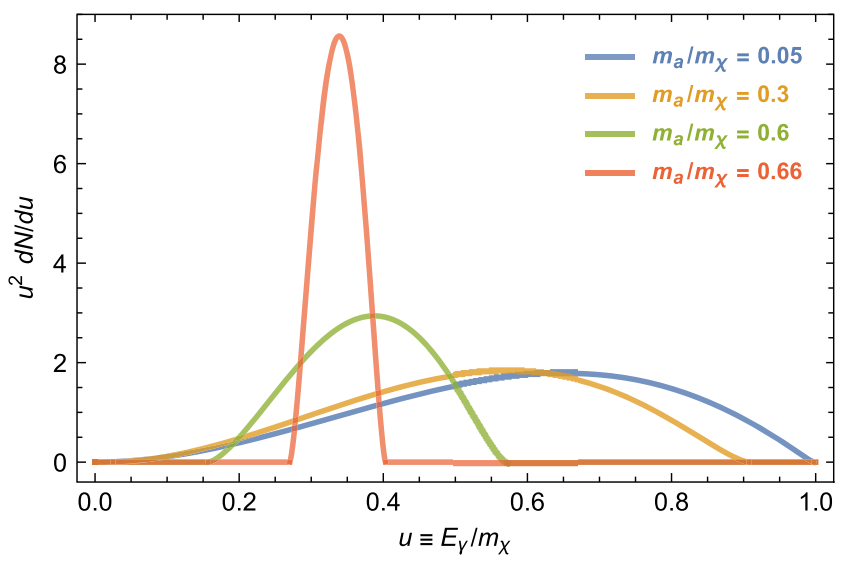

FIG. 4. Photon spectra resulting from $\chi \chi \rightarrow a a a \rightarrow 6 \gamma$ in the HSAP model, for four fiducial values of $\eta=m_{a} / m_{\chi}: 0.05$ (blue), 0.3 (yellow), 0.6 (green), and 0.66 (red). As $\eta$ increases toward the kinematic limit, the restricted phase space forces the spectrum to become increasingly narrow and peaked.

\section{Photon spectra from $a \rightarrow Z \gamma$ and $a \rightarrow Z Z$ decays}

Once $m_{a}>m_{Z}\left(2 m_{Z}\right)$, the decay channel $a \rightarrow Z \gamma$ $(a \rightarrow Z Z)$ opens up. These decay channels result in a continuum of numerous but lower-energy photons from hadron decays, as well as final state radiation off of charged leptons. To obtain photon spectra $d N / d E$ for DM annihilation channels with any number of final-state $Z$ bosons, we proceed numerically. A Monte Carlo sampling method was employed to compute the four-momenta of $a$ particles produced in $\chi \chi \rightarrow a a a$ annihilations following the nonrelativistic distribution of Eq. (7), as well as the momenta of their daughter photons and $Z$ bosons. The photon spectrum resulting from a $Z$ boson in its rest frame was computed using Pythia8 [39]. The resulting photons were then boosted to the Galactic rest frame.

Figure 5 shows the normalized photon spectra resulting from $a$ decays to $\gamma \gamma$ (yellow), $Z Z$ (blue), and $Z \gamma$ (green) for an example parameter point with $m_{\chi}=1500 \mathrm{GeV}$ and

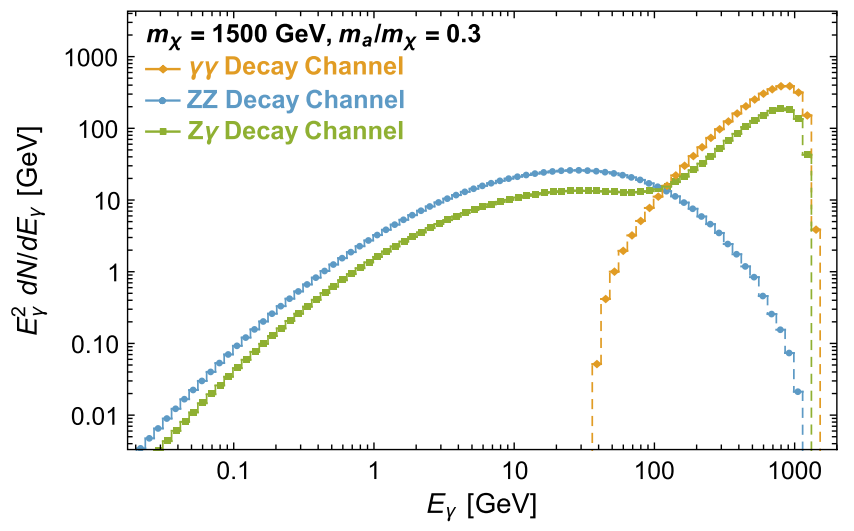

FIG. 5. Photon spectra $E^{2} d N / d E$ resulting from $a$ decays to $\gamma \gamma$ (yellow), $Z Z$ (blue), and $Z \gamma$ (green). We fix $m_{\chi}=1500 \mathrm{GeV}$ and $\eta=0.3$. 
$\eta=0.3$. The $\gamma \gamma$ decay process produces comparatively more high-energy photons with a narrower distribution. The spectrum from $Z Z$ decay is considerably broader with a smooth low-energy tail. The $Z \gamma$ decay spectrum inherits features from both $\gamma \gamma$ and $Z Z$ spectra, albeit with a slightly smaller maximum photon energy than the $\gamma \gamma$ spectrum due to the nonzero $m_{Z}$.

\section{THE ASTROPHYSICAL MODEL}

Following $[17,27,40]$, we consider a simple parametric model describing possible DM density spikes at the Galactic center. We take the DM halo to be described by a generalized Navarro-Frenk-White (NFW) model, which in the central regions of the Galaxy, i.e., well within the scale radius, can be described by a power law $\rho(r)=\rho\left(r_{0}\right)\left(r / r_{0}\right)^{\gamma_{\mathrm{c}}}$. We anchor this power law to the Solar System, where we take the local density of DM to be $\rho\left(r_{\odot}\right)=0.3 \mathrm{GeV} / \mathrm{cm}^{3}$ [41], at a distance $r_{\odot}=8.46 \mathrm{kpc}$ from the Galactic center [42]. DM-only simulations typically yield values of the cusp exponent $\gamma_{c}$ within the range $0.9 \lesssim \gamma_{c} \lesssim 1.2[43,44]$, while the adiabatic contraction of the central halo following baryonic collapse can lead to larger values of $\gamma_{c}$ [45-47]. We will treat $\gamma_{c}$ as a free parameter.

The Milky Way's SMBH has mass $M=4 \times 10^{6} M_{\odot}$ $[48,49]$. Its gravitational zone of influence extends out to the radius $r_{h} \equiv G M / v_{0}^{2}$, where the gravitational potential energy due to the $\mathrm{BH}$ is equal to the typical kinetic energy of a DM particle in the halo. Here $v_{0}$ is the velocity dispersion in the inner halo. We adopt as our fiducial dispersion $v_{0}=105 \pm 20 \mathrm{~km} \mathrm{~s}^{-1}$ [50]. Numerical studies indicate that the spike itself begins growing somewhat within $r_{h}$, at $r_{b}=0.2 r_{h}$ [30,51]. The spike is also welldescribed as a power law, $\rho_{\mathrm{sp}}(r)=\rho\left(r_{b}\right)\left(r / r_{b}\right)^{\gamma_{\mathrm{sp}}}$, although the spike index $\gamma_{\mathrm{sp}}$ depends sensitively on the formation history of the SMBH and the properties of its environment. Spikes that form around a $\mathrm{BH}$ that grows adiabatically at the center of a cuspy DM halo are very steep, $\gamma_{\mathrm{sp}}=$ $\left(9-2 \gamma_{c}\right) /\left(4-\gamma_{c}\right)$ [23]; on the other hand, if the $\mathrm{BH}$ is not at the dynamical center of its halo, then it produces a very shallow spike, $\gamma_{\mathrm{sp}}=1 / 2[28,29]$. The dynamical heating of DM from gravitational interactions with a dense and cuspy stellar distribution results in a spike solution with limiting index $\gamma_{\mathrm{sp}}=1.5$, attained when the system has fully equilibrated [30-32,52]. Nonequilibrated spikes with intermediate power laws are possible if the DM at the Galactic center is still in the process of equilibrating [30]. ${ }^{1}$ In order to succinctly capture the range of signal strengths predicted

\footnotetext{
${ }^{1}$ An alternate parametrization of nonequilibrated spikes undergoing a baryonic heating process, developed in [53], models the reduction of the spike signal through modifications of $r_{b}$ rather than $\gamma_{\mathrm{sp}}$. Both approaches give indistinguishable results for the spike signal [54].
}

by these various different spike formation and evolution scenarios, we treat $\gamma_{\mathrm{sp}}$ as a free parameter and vary it between 1.5 and its adiabatic value.

Once the DM density in the spike reaches the value $\rho_{\text {ann }}=m_{\chi} /(\langle\sigma v\rangle \tau)$, where $\tau \approx 10^{10} \mathrm{yr}$ is the lifetime of the spike, DM annihilations become rapid enough to deplete the spike. We define the radius at which this occurs as $r_{\text {in }}=r_{b} \cdot\left(\rho_{b} / \rho_{\text {ann }}\right)^{1 / \gamma_{\text {sp }}}$. For $r<r_{\text {in }}$, the spike follows a very mild power law, $\rho(r) \propto r^{-1 / 2}$ in the case of $s$-wave annihilations $[55,56]$. Finally the inner boundary of the spike is obtained at $r<4 G M$ [57]. Altogether, then, we take for our model,

$$
\begin{aligned}
\rho(r) & =0 \quad(r<4 G M), \\
& =\frac{\rho_{\mathrm{sp}}(r) \rho_{\text {in }}(r)}{\rho_{\mathrm{sp}}(r)+\rho_{\text {in }}(r)} \quad\left(4 G M \leq r<r_{b}\right), \\
& =\rho_{b}\left(r_{b} / r\right)^{\gamma_{c}} \quad\left(r_{b} \leq r<r_{\odot}\right),
\end{aligned}
$$

where $\rho_{\mathrm{sp}}(r)=\rho_{b}\left(r_{b} / r\right)^{\gamma_{\mathrm{sp}}}, \rho_{\mathrm{in}}(r)=\rho_{\mathrm{ann}}\left(r_{\text {in }} / r\right)^{0.5}, \rho_{b}=$ $\rho\left(r_{\odot}\right) \cdot\left(r_{\odot} / r_{b}\right)^{\gamma_{\mathrm{c}}}$, and we have defined a spike profile that smoothly interpolates between the inner spike with index $1 / 2$ and the outer spike with index $\gamma_{\mathrm{sp}}$.

With the parameter values adopted here, $r_{b} \approx 0.3 \mathrm{pc}$, while for typical DM parameters in the HSAP model the radius $r_{\text {in }} \sim$ few $\times 10^{-5} \mathrm{pc}$. The inner spike structure, which dominates the emission, then subtends $\sim 4$ milliarcsec on the sky, which is several orders of magnitude below the typical resolution of the Fermi telescope. We thus treat the spike signal as a point source. The differential photon flux observed on Earth from the spike is then given by

$$
\frac{d \Phi_{\mathrm{sp}}}{d E_{\gamma}}=\frac{1}{r_{\odot}^{2}} \int_{4 G M}^{r_{\mathrm{b}}} d r \rho(r)^{2} r^{2} \frac{\langle\sigma v\rangle}{2 m_{\chi}^{2}} \frac{d N}{d E_{\gamma}} .
$$

However, the spike sits on top of a bright halo, which can also contribute to the observed central signal as

$$
\frac{d \Phi}{d E_{\gamma}}=\int_{\Delta \Omega} d \Omega \int_{\text {l.o.s. }} \rho(r)^{2} \frac{1}{4 \pi} \frac{\langle\sigma v\rangle}{2 m_{\chi}^{2}} \frac{d N}{d E_{\gamma}} d \ell,
$$

where the line-of-sight (l.o.s.) distance $\ell$ at an angle $\theta$ away from the $\mathrm{GC}$ is given by

$$
r=\sqrt{\ell^{2}+r_{\odot}^{2}-2 \ell r_{\odot} \cos \theta} .
$$

We conservatively include the emission from the central region of the halo, using an energy-dependent half-angle for Fermi and $0.5^{\circ}$ for H.E.S.S., and indicate where this halo emission, $\Phi_{\text {halo }}$, exceeds the contribution from the spike, $\Phi_{\mathrm{sp}}$ in the dominant energy bin. Our main interest is in the regime where the spike dominates, $\Phi_{\mathrm{sp}} \gg \Phi_{\text {halo }}$. This choice allows us to succinctly account for both the finite 
angular resolution of gamma-ray telescopes, notably Fermi [58], and demarcate the region where we might expect challenges from resolving a point source on top of a bright and nonuniform background. To account for Fermi's energy-dependent angular resolution, we consider the halo flux within a cone with half-angle given by the average $68 \%$ containment angle as a function of photon energy, which ranges between $5.3^{\circ}$ for $100 \mathrm{MeV}$ photons and $0.1^{\circ}$ for $100 \mathrm{GeV}$ photons [58]. In plotting spike spectra and evaluating limits, in each bin we adopt an effective angular resolution using the $68 \%$ containment angle of the average photon energy in that bin, as determined from the $\gamma \gamma$ energy spectrum, Eq. (23).

\section{SENSITIVITY TO FAINT $s$-WAVE ANNIHILATION SIGNALS}

Both Fermi and H.E.S.S. have observed bright point sources in the Galactic center that the respective collaborations have associated with $\mathrm{Sgr} \mathrm{A}^{*}$. The Fermi point source 3FGL J1745.6-2859 C is observed in gamma rays with $100 \mathrm{MeV}<E_{\gamma}<100 \mathrm{GeV}$ [59], while the H.E.S.S. source HESS J1745-290 is observed in gamma rays with $180 \mathrm{GeV}<E_{\gamma}<79 \mathrm{TeV}$ [60]. We show the observed spectra of these point sources in the solid histograms of Fig. 6. The abrupt decrease in binned flux magnitude starting at $m_{\chi}=180 \mathrm{GeV}$ reflects the different bin sizes in the two experiments. The energy gap between Fermi and H.E.S.S. data sets at $100<m_{\chi}<180 \mathrm{GeV}$ is clearly visible. For comparison, the dashed histograms show four example predictions for the primary photon spectra arising from HSAP DM annihilations within DM density spikes. We show predictions for four different

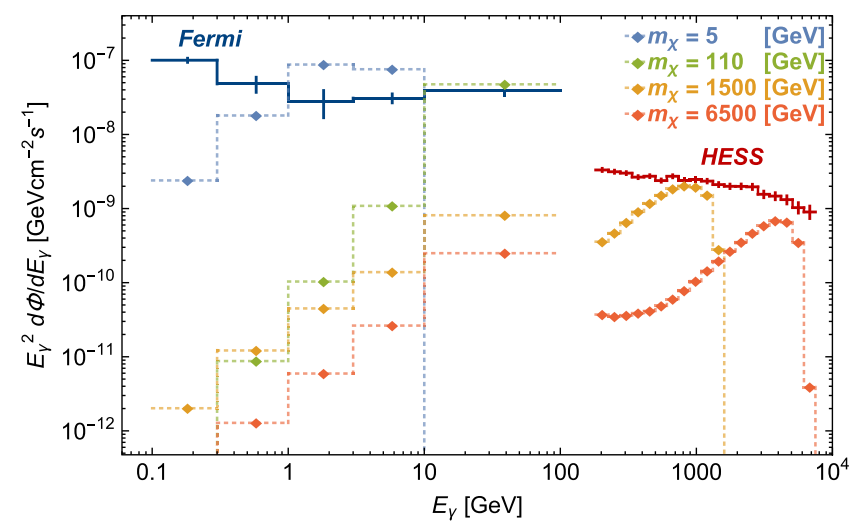

FIG. 6. Observed GC $\gamma$-ray point source spectra compared with example model predictions. The spectra of Fermi source 3FGL J1745.6-2859C (dark blue) [59] and H.E.S.S. source HESS J1745-290 (dark red) [60] associated with Sgr A* are plotted in solid. The dashed histograms are four predicted flux spectra for $m_{\chi}=5 \mathrm{GeV}$ (light blue), 110 (green), 1500 (yellow), and $6500 \mathrm{GeV}$ (orange). We fix $\gamma_{c}=1.2, \gamma_{\mathrm{sp}}=1.8$, and $m_{a} / m_{\chi}=0.1$. values of DM mass at fixed $\eta=0.1, \gamma_{c}=1.2$, and $\gamma_{\mathrm{sp}}=1.8$.

To estimate sensitivity to HSAP DM annihilations within $\mathrm{BH}$ spikes, we use the simple criterion that the spike flux should not exceed the observed flux from either $3 \mathrm{FGL}$ J1745.6-2859C or HESS J1745-290 at more than 95\% C.L. in any bin. Figure 7 shows the values of the $s$-wave annihilation cross section excluded by this procedure for six fixed values of $m_{\chi}$ in two different astrophysical scenarios. In this figure we take $\operatorname{Br}(a \rightarrow \gamma \gamma)=1$ throughout, so that the photon spectrum is given by Eq. (23). Visible kinks in the lines reflect when the peak of the photon spectrum moves from one bin to another as $\eta$ changes. For $m_{\chi}=5,11$, and $50 \mathrm{GeV}$, the constraints come from Fermi, while for $m_{\chi}=750,1500$, and $6500 \mathrm{GeV}$, the constraints come from H.E.S.S. The improvement of the limits as $\eta \rightarrow 2 / 3$ reflects the increasing sharpness of the peak in the photon spectrum. From Fig. 7, we see that adiabatic spikes are sensitive to cross sections some 4
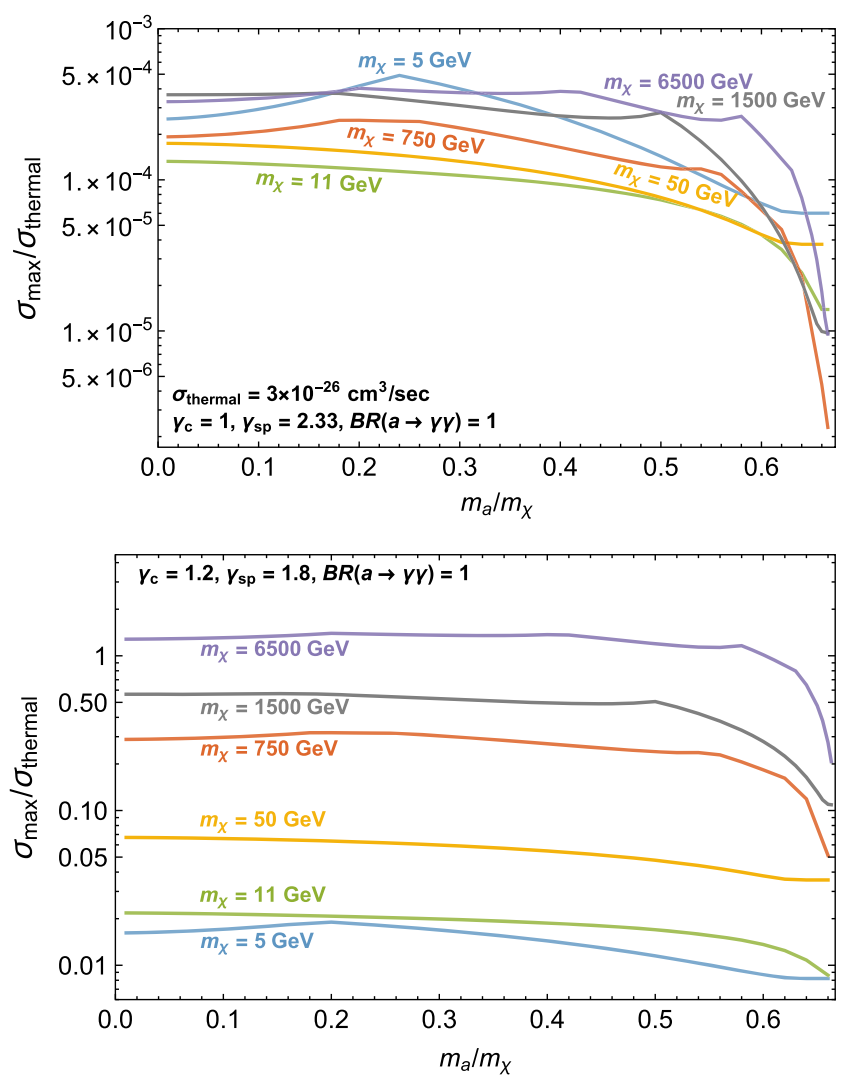

FIG. 7. Value of the $s$-wave cross section, as a fraction of the reference thermal value $\sigma_{\text {thermal }} \equiv 3 \times 10^{-26} \mathrm{~cm}^{3} / \mathrm{s}$, for which the photon flux from a BH spike exceeds either Fermi or H.E.S.S. observations at $95 \%$ C.L. in at least one bin. Results are shown as a function of the mass ratio $\eta=m_{a} / m_{\chi}$ for six fixed values $m_{\chi}$. In the top panel we fix $\gamma_{c}=1$ and consider the adiabatic $\gamma_{\mathrm{sp}}=2.33$, and in the bottom panel we use $\gamma_{c}=1.2$ and $\gamma_{\mathrm{sp}}=1.8$. We take $\operatorname{Br}(a \rightarrow \gamma \gamma)=1$. 

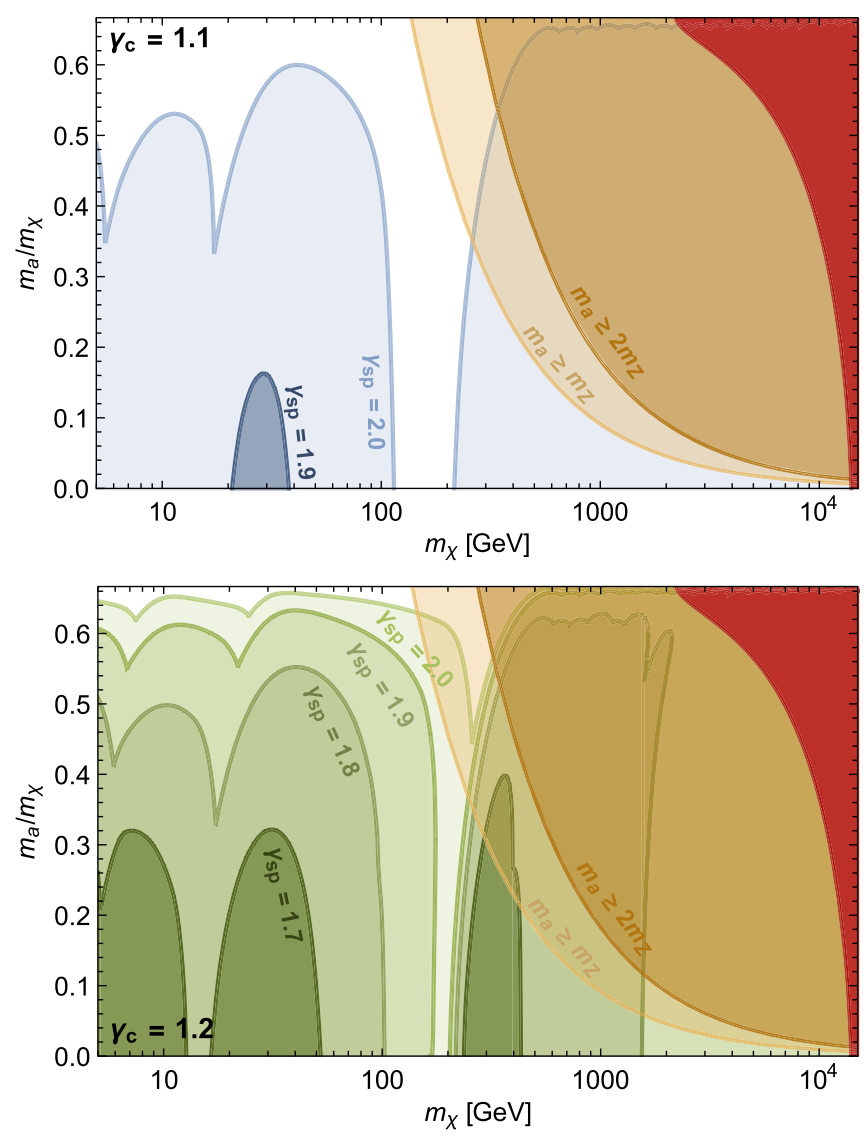

FIG. 8. Excluded regions in $m_{a}-m_{\chi}$ parameter space for fixed $\gamma_{\mathrm{c}}, \gamma_{\mathrm{sp}}$. The top panel fixes $\gamma_{c}=1.1$ and $\gamma_{\mathrm{sp}}=1.9,2.0$; the bottom takes $\gamma_{c}=1.2$ and $\gamma_{\mathrm{sp}}=1.7,1.8,1.9,2.0$. We take $\operatorname{Br}(a \rightarrow \gamma \gamma)=1$ throughout. The $a \rightarrow Z \gamma(a \rightarrow Z Z)$ decay channel is available in the light (dark) tan shaded regions. The dark red region indicates where $y^{2} / 4 \pi \geq 1$.

orders of magnitude smaller than the standard thermal target, while for more moderate values of $\gamma_{\mathrm{sp}}$, the resulting sensitivity even in relatively cuspy haloes is less dramatic but still interesting. Constraints on the cross section for higher mass DM are generally weaker; however, in the HSAP model studied in this paper, this effect is substantially offset by the larger predicted $s$-wave cross sections at high mass.

Figure 8 shows excluded regions of $m_{\chi}-\eta$ parameter space for fixed choices of spike and halo indices. The top panel fixes $\gamma_{c}=1.1$ together with $\gamma_{\mathrm{sp}}=1.9,2.0$ (no parameter space is excluded for $\gamma_{\mathrm{sp}} \leq 1.8$ ). The bottom panel has $\gamma_{\mathrm{c}}=1.2$ with $\gamma_{\mathrm{sp}}=1.7,1.8,1.9,2.0$. For each exclusion boundary, a concave curve segment corresponds to the exclusion given by one energy bin from either the Fermi or H.E.S.S. point source. As expected from Fig. 3, we see strong variation of the sensitivity with the mass ratio $\eta$ at small $m_{\chi}$.

In Fig. 8 we continue to take for simplicity $\operatorname{Br}(a \rightarrow \gamma \gamma)=1$, as this case can be handled analytically. We indicate with the shaded light (dark) tan area where

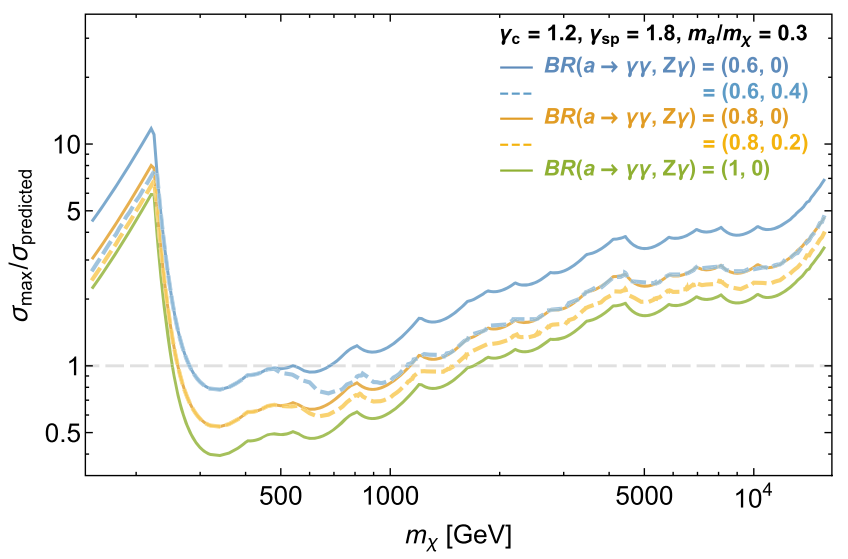

FIG. 9. Dependence of the maximum allowed $s$-wave annihilation cross section on the DM annihilation channels. We study limits for fixed $\{\operatorname{Br}(a \rightarrow \gamma \gamma), \operatorname{Br}(a \rightarrow Z \gamma)\}=\{1,0\},\{0.8,0.2\}$, and $\{0.6,0.4\}$ in blue, yellow, and green respectively. Solid lines use only photons from the $\gamma \gamma$ channel, while dashed lines use all photons. Results are shown for an example point with $\gamma_{c}=1.2$, $\gamma_{\mathrm{sp}}=1.8$, and $\eta=0.3$.

$m_{a}>m_{Z}\left(2 m_{Z}\right)$, and this assumption breaks down. In general, once $Z$ bosons are kinematically accessible, the details of the DM annihilation spectrum will depend on the modeling choice of how the pseudoscalar $a$ couples to SM electroweak bosons. However it is straightforward to translate the results of Fig. 8 to the nonzero branching fractions into $Z \gamma$ and $Z Z$ predicted by Eq. (14) in most of parameter space, as we now discuss.

Sensitivity to DM annihilations in the HSAP model is dominated by the peak of the photon spectrum in the $\gamma \gamma$ and $Z \gamma$ channels; the continuum photons coming from $Z$ bosons are not important for determining the sensitivity. This can be seen from Fig. 9, where we compare limits set using only $\gamma \gamma$ decays to those determined from both $\gamma \gamma$ and $Z \gamma$ decays. This figure shows the maximum $s$-wave annihilation cross section allowed by observations of the GC, relative to the prediction of the HSAP model, as a function of DM mass, and demonstrates how the limit changes as the branching fractions of $a$ are varied. Green, yellow, and blue curves show exclusions assuming branching ratios $\{\operatorname{Br}(a \rightarrow \gamma \gamma), \operatorname{Br}(a \rightarrow Z \gamma)\}=\{1,0\}, \quad\{0.8,0.2\}$, and $\{0.6,0.4\}$ respectively. The solid curves use only photons from the $\gamma \gamma$ channel to set a limit, while the dashed curves show results from including all photons. ${ }^{2}$

Figure 9 restricts attention to the range of DM masses where $Z$ bosons are kinematically accessible for the chosen value of $\eta=0.3$. The sharp feature near $m_{\chi} \sim 200 \mathrm{GeV}$ reflects the transition from Fermi to H.E.S.S. In the high-

\footnotetext{
${ }^{2}$ This discussion is sensible for the SM couplings adopted in this work, where the maximum value of $\operatorname{Br}(a \rightarrow Z Z)$ is $\approx 12 \%$. In models where $\operatorname{Br}(a \rightarrow \gamma \gamma)$ is sufficiently suppressed compared to continuum decay modes that the continuum, rather than the peak, dominates the limits, this argument will no longer apply.
} 
$m_{\chi}$ region, where the peak of the photon spectrum is fully within H.E.S.S.' energy range, the difference in the peak energies of the $\gamma \gamma$ and $Z \gamma$ spectra is small compared to the peak energy. Thus in the high- $m_{\chi}$ regime a limit can be estimated simply from counting the number of primary photons from $a$ decays: i.e., by using the $\gamma \gamma$ limit but multiplying by the factor $\operatorname{Br}(\gamma \gamma)+\frac{1}{2} \operatorname{Br}(Z \gamma)<1$. This is demonstrated in Fig. 9 by the excellent agreement of the blue dashed and orange solid curves at large $m_{\chi}$. Figure 9 also shows the expected linear scaling of the high- $m_{\chi}$ excluded cross section with $\operatorname{Br}(\gamma \gamma)$. By contrast, in the gap between Fermi and H.E.S.S.' observations, the limit is dominated by the high-energy shoulder of the photon spectrum, where photons from $Z \gamma$ decays make a negligible contribution.

As Fig. 8 already demonstrates, observational sensitivity to the HSAP model is strongly dependent on $\gamma_{\mathrm{sp}}$ and especially $\gamma_{c}$. Any astrophysical parameter combination with $\gamma_{c} \geq 1.2$ and $\gamma_{\mathrm{sp}} \geq 2.0$ is almost entirely ruled out. Conversely, very little parameter space is excluded for $\gamma_{c} \leq 1.1$ and $\gamma_{\mathrm{sp}} \leq 1.8$. We now turn to examining the sensitivity as a function of $\gamma_{c}$ and $\gamma_{\mathrm{sp}}$ for fixed particle parameters in Fig. 10. Here we show results for fixed $m_{\chi}=11 \mathrm{GeV}$ (top) and $1500 \mathrm{GeV}$ (bottom), and three fixed choices of mass ratio $\eta$. The gray shaded regions indicate where the contribution from the central $0.5^{\circ}$ of the halo exceeds the contribution from the spike. The exclusions we find are thus indeed driven by the spike for the bulk of parameter space, except at large values of $\gamma_{c}$ where a more careful combined study of the halo plus potential spike point source would be warranted. Canonical adiabatic spikes are almost entirely disfavored, except at very small values of $\gamma_{c}$.

In general, points with $\eta=0.1$ see more stringent limits than those with $\eta=0.3$ and 0.6. This occurs for two main reasons. First, in the HSAP model, the magnitude of the $s$-wave cross section depends sensitively on $\eta$ (see Fig. 3), especially for the relatively low value $m_{\chi}=11 \mathrm{GeV}$ in the top panel. Second, once it is kinematically possible to produce $Z$ bosons, the flux in the photon peak is reduced. The branching fraction $\gamma \gamma+\frac{1}{2} Z \gamma$ is typically larger for smaller values of $\eta$. This second effect is relevant in the bottom panel of Fig. 10, where the pseudoscalar is heavy enough to decay into $Z \gamma(\eta=0.1,0.3)$ and $Z Z(\eta=0.6)$ final states. In this panel we show two sets of limits. The green curves, marked with asterisks, show limits obtained by artificially setting $\operatorname{Br}(\gamma \gamma)=1$, while the blue curves show limits using the branching fractions of Eq. (14). In particular, we set $\{\operatorname{Br}(a \rightarrow \gamma \gamma), \operatorname{Br}(a \rightarrow Z \gamma)\} \approx\{0.65,0.35\}$ for $\eta=0.1, \quad\{\operatorname{Br}(a \rightarrow \gamma \gamma), \operatorname{Br}(a \rightarrow Z \gamma), \operatorname{Br}(a \rightarrow Z Z)\} \approx$ $\{0.32,0.61,0.07\}$ for $\eta=0.3$, and $\{\operatorname{Br}(a \rightarrow \gamma \gamma)$, $\operatorname{Br}(a \rightarrow Z \gamma), \operatorname{Br}(a \rightarrow Z Z)\} \approx\{0.3,0.62,0.08\}$ for $\eta=0.6$. Single asterisks indicate that $a \rightarrow Z \gamma$ is kinematically allowed, while double asterisks indicate both $a \rightarrow Z \gamma$ and $a \rightarrow Z Z$ decay channels are possible.
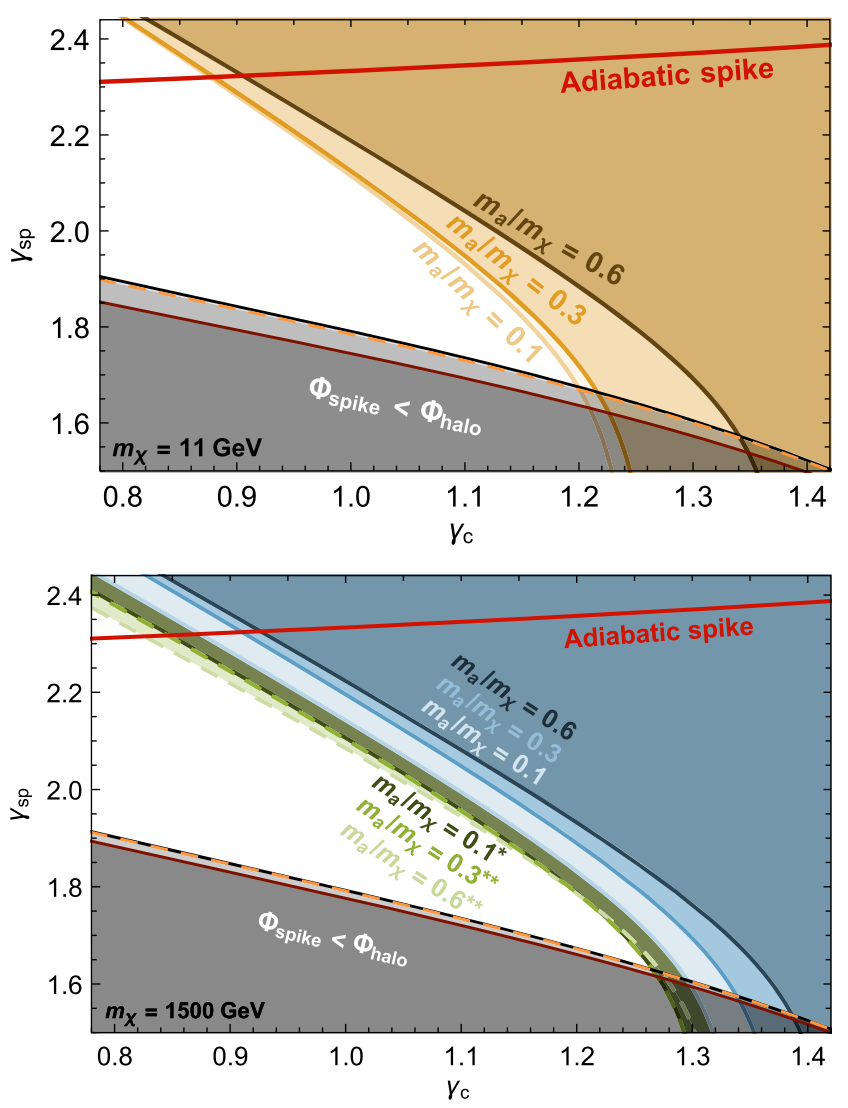

FIG. 10. Exclusions in $\gamma_{c}-\gamma_{\mathrm{sp}}$ parameter space for fixed $m_{\chi}=$ $11 \mathrm{GeV}$ (top) and $1500 \mathrm{GeV}$ (bottom) and $m_{a} / m_{\chi}=0.1,0.3,0.6$. Predictions for adiabatic spikes are indicated by the red line. The gray shaded areas indicate where the flux from the central region of the halo exceeds the flux from the spike in the energy bin that dominates the limits; the three boundary curves show $\Phi_{\text {spike }}=$ $\Phi_{\text {halo }}$ with $m_{a} / m_{\chi}=0.1,0.3$, and 0.6 from top to bottom (black, dashed orange, dark red). In the bottom panel, the blue curves use the branching ratios of Eq. (14), while the green curves, marked with asterisks, artificially assume $\operatorname{Br}(a \rightarrow \gamma \gamma)=1$.

The exclusions for $\eta=0.1$ and 0.3 in the bottom panel of Fig. 10 are set by the same bin, while for $\eta=0.6$ the peak of the photon spectrum has migrated far enough to higher energies that it is instead the neighboring bin that determines the exclusion. This results in the different shapes of the excluded regions obtained for $\eta=0.6$ versus $\eta=0.1$ and 0.3 that can be most easily seen in the green curves. When we incorporate the above branching ratios into $Z \gamma$ and $Z Z$, the flux in the peak of the photon spectrum is reduced and the limits weaken accordingly, but the shapes of the curves remain the same.

\section{SUMMARY AND CONCLUSIONS}

We have performed a detailed study of DM annihilations in a hidden sector axion portal (HSAP) model where fermionic DM $\chi$ annihilates to pseudoscalars $a$. We consider here the regime where both the $p$-wave $\chi \chi \rightarrow a a$ and 
$s$-wave $\chi \chi \rightarrow a a a$ annihilation channels are kinematically available. We find that the relic abundance is determined dominantly by $p$-wave annihilations at low DM masses, while $s$-wave contributions can dominate at higher DM masses. In all cases the $s$-wave cross section dominates the annihilation in the Milky Way today and can be suppressed by as much as $10^{-4}$ relative to the standard expectation for a thermal WIMP. We take the pseudoscalar mediator $a$ to decay to SM states via an axionlike coupling to SM hypercharge gauge bosons. When $m_{a}<m_{Z}$, the pseudoscalar decays exclusively via $a \rightarrow \gamma \gamma$. We derive the photon spectrum resulting from $\chi \chi \rightarrow a a a \rightarrow 6 \gamma$ analytically in the limit of nonrelativistic DM annihilations. This spectrum is dominated by a characteristic high-energy spectral feature that could help to facilitate discovery and identification of a DM signal in the busy environment of the Galactic center. When $m_{a}$ is large enough to allow for $Z \gamma$ and $Z Z$ decays, we determine the spectra for those decay channels numerically.

DM density spikes around the Milky Way's supermassive black hole offer a speculative but irreplaceable potential discovery handle on this model. We estimated current sensitivity to HSAP DM annihilation within such spikes by comparing the predicted pointlike gamma-ray spike signals to Fermi and H.E.S.S. observations of Sgr A*. We find interesting sensitivity to the HSAP model across a wide range of both particle and astrophysical parameters. Canonical adiabatic spikes can probe cross sections some 4 orders of magnitude below the standard thermal target and are accordingly almost entirely ruled out in our model space. However, substantial portions of parameter space remains open for shallower spikes, particularly in less cuspy haloes. In such (perhaps more realistic) astrophysical scenarios, we find that searches for spatially localized, sharp features in the gamma-ray spectrum remain wellmotivated as a potential discovery handle on otherwise challenging models of DM.

The HSAP model compactly provides examples of both parametrically suppressed annihilation cross sections at low DM mass and mass-suppressed annihilation rates at high DM mass. As is common for models of secluded DM [61], the HSAP model also has severely suppressed signals at both direct detection and collider experiments, making indirect detection a vital experimental probe of this model. This HSAP model is only one example of a rich variety of particle models that predict DM annihilation signals substantially below current observational sensitivity; the enhanced sensitivity to such faint annihilation signals in DM density spikes thus remains important to consider even as thermal targets are surpassed.

\section{ACKNOWLEDGMENTS}

We are happy to acknowledge useful conversations with A. Srinivasan. We particularly thank J. Tu for assistance with computing and B. Mandava for assistance in algorithm efficiency improvement. The work of B.T.C. was supported by the Lorella M. Jones and the Philip J. and Betty M. Anthony Undergraduate Research Awards at the University of Illinois at Urbana-Champaign. The work of S. L. S. is supported in part by NSF Grant No. PHY1662211 and NASA Grant No. 80NSSC17K0070. The work of J.S. is supported by DOE CAREER Grant No. DE-SC0017840.
[1] P. A. R. Ade et al. (Planck Collaboration), Planck 2015 results. XIII. Cosmological parameters, Astron. Astrophys. 594, A13 (2016).

[2] T. R. Slatyer, Indirect dark matter signatures in the cosmic dark ages. I. Generalizing the bound on s-wave dark matter annihilation from Planck results, Phys. Rev. D 93, 023527 (2016).

[3] M. Ackermann et al. (Fermi-LAT Collaboration), Searching for Dark Matter Annihilation from Milky Way Dwarf Spheroidal Galaxies with Six Years of Fermi Large Area Telescope Data, Phys. Rev. Lett. 115, 231301 (2015).

[4] A. Albert et al. (Fermi-LAT and DES Collaborations), Searching for dark matter annihilation in recently discovered Milky Way satellites with Fermi-LAT, Astrophys. J. 834, 110 (2017).

[5] J. L. Feng, H. Tu, and H.-B. Yu, Thermal relics in hidden sectors, J. Cosmol. Astropart. Phys. 10 (2008) 043.

[6] J. A. Evans, C. Gaidau, and J. Shelton, Leak-in dark matter, J. High Energy Phys. 01 (2020) 032.
[7] N. Fornengo, A. Riotto, and S. Scopel, Supersymmetric dark matter and the reheating temperature of the universe, Phys. Rev. D 67, 023514 (2003).

[8] G. Gelmini, P. Gondolo, A. Soldatenko, and C. E. Yaguna, The effect of a late decaying scalar on the neutralino relic density, Phys. Rev. D 74, 083514 (2006).

[9] D. Hooper, Is the CMB telling us that dark matter is weaker than weakly interacting?, Phys. Rev. D 88, 083519 (2013).

[10] K. Griest and D. Seckel, Three exceptions in the calculation of relic abundances, Phys. Rev. D 43, 3191 (1991).

[11] R. T. D'Agnolo and J. T. Ruderman, Light Dark Matter from Forbidden Channels, Phys. Rev. Lett. 115, 061301 (2015).

[12] J. Kopp, J. Liu, T. R. Slatyer, X.-P. Wang, and W. Xue, Impeded dark matter, J. High Energy Phys. 12 (2016) 033.

[13] A. Delgado, A. Martin, and N. Raj, Forbidden dark matter at the weak scale via the top portal, Phys. Rev. D 95, 035002 (2017).

[14] H. Goldberg, Constraint on the Photino Mass from Cosmology, Phys. Rev. Lett. 50, 1419 (1983). 
[15] K. Griest, Cross-sections, relic abundance and detection rates for neutralino dark matter, Phys. Rev. D 38, 2357 (1988).

[16] C. Boehm and P. Fayet, Scalar dark matter candidates, Nucl. Phys. B683, 219 (2004).

[17] J. Shelton, S. L. Shapiro, and B. D. Fields, Black Hole Window Into $p$-Wave Dark Matter Annihilation, Phys. Rev. Lett. 115, 231302 (2015).

[18] J. A. Evans, S. Gori, and J. Shelton, Looking for the WIMP next door, J. High Energy Phys. 02 (2018) 100.

[19] M. Cirelli, P. Panci, G. Servant, and G. Zaharijas, Consequences of DM/antiDM oscillations for asymmetric WIMP dark matter, J. Cosmol. Astropart. Phys. 03 (2012) 015.

[20] M. J. Baker et al., The coannihilation codex, J. High Energy Phys. 12 (2015) 120.

[21] M. Garny, J. Heisig, B. Llf, and S. Vogl, Coannihilation without chemical equilibrium, Phys. Rev. D 96, 103521 (2017).

[22] R. T. D’Agnolo, D. Pappadopulo, and J. T. Ruderman, Fourth Exception in the Calculation of Relic Abundances, Phys. Rev. Lett. 119, 061102 (2017).

[23] P. Gondolo and J. Silk, Dark Matter Annihilation at the Galactic Center, Phys. Rev. Lett. 83, 1719 (1999).

[24] M. A. Amin and T. Wizansky, Relativistic dark matter at the Galactic Center, Phys. Rev. D 77, 123510 (2008).

[25] M. Cannoni, M. E. Gomez, M. A. Perez-Garcia, and J. D. Vergados, New gamma ray signal from gravitationally boosted neutralinos at the Galactic Center, Phys. Rev. D 85, 115015 (2012).

[26] T. Lacroix, C. Bhm, and J. Silk, Ruling out thermal dark matter with a black hole induced spiky profile in the M87 galaxy, Phys. Rev. D 92, 043510 (2015).

[27] C. Johnson, R. Caputo, C. Karwin, S. Murgia, S. Ritz, and J. Shelton, Search for gamma-ray emission from $p$-wave dark matter annihilation in the Galactic Center, Phys. Rev. D 99, 103007 (2019).

[28] T. Nakano and J. Makino, On the cusp around central black holes in luminous elliptical galaxies, Astrophys. J. 525, L77 (1999).

[29] P. Ullio, H. Zhao, and M. Kamionkowski, A dark matter spike at the Galactic Center?, Phys. Rev. D 64, 043504 (2001).

[30] D. Merritt, Evolution of the Dark Matter Distribution at the Galactic Center, Phys. Rev. Lett. 92, 201304 (2004).

[31] O. Y. Gnedin and J. R. Primack, Dark Matter Profile in the Galactic Center, Phys. Rev. Lett. 93, 061302 (2004).

[32] D. Merritt, S. Harfst, and G. Bertone, Collisionally regenerated dark matter structures in Galactic nuclei, Phys. Rev. D 75, 043517 (2007).

[33] S. L. Shapiro and V. Paschalidis, Self-interacting dark matter cusps around massive black holes, Phys. Rev. D 89, 023506 (2014).

[34] E. Kolb and M. Turner, The Early Universe, Frontiers in Physics (Addison-Wesley, Reading, Massachusetts, 1990).

[35] G. Bertone, G. Sigl, and J. Silk, Annihilation radiation from a dark matter spike at the Galactic Center, Mon. Not. R. Astron. Soc. 337, 98 (2002).

[36] R. Aloisio, P. Blasi, and A. V. Olinto, Neutralino annihilation at the Galactic Center revisited, J. Cosmol. Astropart. Phys. 05 (2004) 007.
[37] M. Regis and P. Ullio, Multi-wavelength signals of dark matter annihilations at the Galactic Center, Phys. Rev. D 78, 043505 (2008).

[38] I. Cholis, D. Hooper, and T. Linden, A critical reevaluation of radio constraints on annihilating dark matter, Phys. Rev. D 91, 083507 (2015).

[39] T. Sjostrand, S. Mrenna, and P.Z. Skands, A brief introduction to PYTHIA 8.1, Comput. Phys. Commun. 178, 852 (2008).

[40] B. D. Fields, S. L. Shapiro, and J. Shelton, Galactic Center Gamma-Ray Excess from Dark Matter Annihilation: Is There A Black Hole Spike?, Phys. Rev. Lett. 113, 151302 (2014).

[41] J. Bovy and S. Tremaine, On the local dark matter density, Astrophys. J. 756, 89 (2012).

[42] T. Do, G. D. Martinez, S. Yelda, A. M. Ghez, J. Bullock, M. Kaplinghat, J. R. Lu, A. H. G. Peter, and K. Phifer, 3D stellar kinematics at the Galactic Center: Measuring the nuclear star cluster spatial density profile, black hole mass, and distance, Astrophys. J. 779, L6 (2013).

[43] J. Diemand, M. Kuhlen, P. Madau, M. Zemp, B. Moore, D. Potter, and J. Stadel, Clumps and streams in the local dark matter distribution, Nature (London) 454, 735 (2008).

[44] J. F. Navarro, A. Ludlow, V. Springel, J. Wang, M. Vogelsberger, S. D. M. White, A. Jenkins, C. S. Frenk, and A. Helmi, The diversity and similarity of cold dark matter halos, Mon. Not. R. Astron. Soc. 402, 21 (2010).

[45] G. R. Blumenthal, S. M. Faber, R. Flores, and J. R. Primack, Contraction of dark matter Galactic halos due to baryonic infall, Astrophys. J. 301, 27 (1986).

[46] O. Y. Gnedin, A. V. Kravtsov, A. A. Klypin, and D. Nagai, Response of dark matter halos to condensation of baryons: Cosmological simulations and improved adiabatic contraction model, Astrophys. J. 616, 16 (2004).

[47] M. Gustafsson, M. Fairbairn, and J. Sommer-Larsen, Baryonic pinching of Galactic dark matter haloes, Phys. Rev. D 74, 123522 (2006).

[48] R. Genzel, F. Eisenhauer, and S. Gillessen, The Galactic Center massive black hole and nuclear star cluster, Rev. Mod. Phys. 82, 3121 (2010).

[49] A. M. Ghez et al., Measuring distance and properties of the Milky Way's central supermassive black hole with stellar orbits, Astrophys. J. 689, 1044 (2008).

[50] K. Gultekin et al., The M-sigma and M-L relations in Galactic bulges and determinations of their intrinsic scatter, Astrophys. J. 698, 198 (2009).

[51] D. Merritt, Single and binary black holes and their influence on nuclear structure, in Carnegie Observatories Centennial Symposium. 1. Coevolution of Black Holes and Galaxies Pasadena, California, 2002 (2003), arXiv:astro$\mathrm{ph} / 0301257$.

[52] G. Bertone and D. Merritt, Time-dependent models for dark matter at the Galactic Center, Phys. Rev. D 72, 103502 (2005).

[53] E.-J. Ahn, G. Bertone, and D. Merritt, Impact of astrophysical processes on the gamma-ray background from dark matter annihilations, Phys. Rev. D 76, 023517 (2007).

[54] P. Sandick, K. Sinha, and T. Yamamoto, Black holes, dark matter spikes, and constraints on simplified models with t-channel mediators, Phys. Rev. D 98, 035004 (2018).

[55] E. Vasiliev, Dark matter annihilation near a black hole: Plateau vs. weak cusp, Phys. Rev. D 76, 103532 (2007). 
[56] S. L. Shapiro and J. Shelton, Weak annihilation cusp inside the dark matter spike about a black hole, Phys. Rev. D 93, 123510 (2016).

[57] L. Sadeghian, F. Ferrer, and C. M. Will, Dark matter distributions around massive black holes: A general relativistic analysis, Phys. Rev. D 88, 063522 (2013).

[58] https://www.slac.stanford.edu/exp/glast/groups/canda/lat_ Performance.htm.
[59] F. Acero et al. (Fermi-LAT Collaboration), Fermi large area telescope third source catalog, Astrophys. J. Suppl. Ser. 218, 23 (2015).

[60] A. Abramowski et al. (H.E.S.S. Collaboration), Acceleration of petaelectronvolt protons in the Galactic Centre, Nature (London) 531, 476 (2016).

[61] M. Pospelov, A. Ritz, and M. B. Voloshin, Secluded WIMP dark matter, Phys. Lett. B 662, 53 (2008). 